\title{
Comorbilidad afectiva en personas con hipertensión arterial: un estudio de caso-control en adultos dominicanos
}

\author{
Zoilo Emilio García-Batista; Kiero Guerra-Peña; Antonio Cano-Vindel; \\ Solmary Xiomara Herrera-Martínez; Pablo Ezequiel Flores-Kanter; Leonardo Adrián Medrano
}

How to cite this article:

García-Batista, Z.E., Guerra-Peña, K., Cano-Vindel, A., Herrera-Martínez, S.X., Flores-Kanter, P.E. \& Medrano, L.A. (2020). Affective comorbidity in patients with hypertension: a case-control study on adults in the Dominican Republic. Acta Colombiana de Psicología, 23(1), 193-204. doi: http://www.doi.org/10.14718/ACP.2020.23.1.10

Recibido, febrero 13/2019; Concepto de evaluación, abril 1/2019; Aceptado, mayo 25/2019

\author{
Zoilo Emilio García-Batista* \\ Pontificia Universidad Católica Madre y Maestra, Santiago de los Caballeros, República Dominicana \\ ORCID: https://orcid.org/0000-0002-0353-4804 \\ Kiero Guerra-Peña \\ Pontificia Universidad Católica Madre y Maestra, Santiago de los Caballeros, República Dominicana \\ ORCID: https://orcid.org/0000-0003-3315-9459 \\ Antonio Cano-Vindel \\ Universidad Complutense de Madrid, Madrid, España \\ ORCID: https://orcid.org/0000-0002-5449-5454 \\ Solmary Xiomara Herrera-Martínez \\ Universidad Arturo Michelena, Valencia, Venezuela \\ ORCID: https://orcid.org/0000-0002-9353-8277 \\ Pablo Ezequiel Flores-Kanter \\ Universidad Siglo 21, Córdoba, Argentina \\ ORCID: https://orcid.org/0000-0002-6712-779X \\ Leonardo Adrián Medrano \\ Pontificia Universidad Católica Madre y Maestra, Santiago de los Caballeros, República Dominicana \\ ORCID: https://orcid.org/0000-0002-3371-5040
}

\begin{abstract}
Resumen
En la literatura científica se encuentra un gran número de controversias y resultados dispares a la hora de explicar la relación entre la hipertensión arterial y algunas variables vinculadas a la afectividad negativa, como la depresión, la ansiedad y la ira. Teniendo esto en cuenta, en la presente investigación se planteó como objetivo principal analizar medidas de ansiedad, depresión e ira en personas adultas que padecen de hipertensión arterial (HTA). A partir de un diseño de caso-control, se contrastaron dos grupos, uno conformado por personas con HTA $(n=50)$ y otro de control equiparado en número de casos, edad y sexo. A nivel general, el grupo de HTA mostró mayores niveles de depresión — en su dimensión somática— y de ira-rasgo — en su dimensión de temperamento- Asimismo, las personas con HTA presentaron síntomas de irritabilidad y pérdida de energía — síntomas depresivo-somáticos—, además de que tendían a enojarse con facilidad o rápidamente — síntomas de temperamento de ira- El abordaje de estos factores psicológicos comórbidos resulta de relevancia dado que en estudios precedentes ha demostrado aumentar la adherencia al tratamiento médico en pacientes que han sido diagnosticados con hipertensión o patologías similares.

Palabras clave: hipertensión, depresión, ansiedad, ira, comorbilidad.
\end{abstract}

\footnotetext{
Santiago de los Caballeros, República Dominicana. C. P.: 51000. zgarcia@pucmm.edu.do
} 


\title{
Affective comorbidity in patients with bypertension: a case-control study on adults in the Dominican Republic
}

\begin{abstract}
Research on the relationship between hypertension and variables linked to negative affectivity has given rise to divergent findings and differing interpretations. The main objective of this paper was therefore to shed light on the issue by analyzing measures of depression, anxiety and anger in adults suffering from hypertension (HTN). Based on a case-control design, a group of individuals suffering from $\operatorname{HTN}(n=50)$ was compared with a control group of the same number of cases, age and sex. In general, the HTN Group showed higher levels of depression in its somatic dimension and of trait anger in its temperament dimension. Individuals with HTN presented symptoms of irritability and loss of energy (depressive-somatic symptoms) and propensity to anger easily (temperament anger symptoms). It has been demonstrated in preceding researches that addressing these comorbid psychological factors is of importance in increase adherence to medical treatment in patients who have been diagnosed with hypertension or similar pathologies.

Key words: hypertension; depression; anxiety; anger; comorbidity.
\end{abstract}

\section{Introducción}

La hipertensión arterial (HTA) es una condición médica crónica considerada como una de las enfermedades de mayor morbilidad y mortalidad en todo el mundo (Wang et al., 2016), además de ser uno de los factores de riesgo más importantes en el surgimiento de diversas patologías cardiovasculares - incluidas las fallas y ataques cardíacos-, y presentar una prevalencia que conlleva altos costos económicos (Wu et al., 2018). Lamentablemente, se estima que su tasa de prevalencia a nivel mundial aumentará hasta 1.56 millones de personas para el 2025 (Organización Mundial de la Salud [OMS], 2000), y se han encontrado pocos estudios acerca de la prevalencia de esta enfermedad en países en vías de desarrollo (Ploth et al., 2018).

Dada la importancia de la HTA y sus implicancias en el sistema de salud, el estudio de los factores de riesgo asociados a ella continúa siendo un tema de investigación y debate (Redina \& Markel, 2018), sobre todo porque en la mayoría de los casos se desconocen las causas que originan las elevaciones crónicas de la presión arterial (p. ej., HTA primaria o esencial; OMS, 2013). Sin embargo, en los últimos años, además de las variables biológicas clásicas, como la obesidad y la edad, los factores afectivos han cobrado una importante relevancia - aunque no suficientemente esclarecida - en la explicación del desarrollo y mantenimiento de la hipertensión esencial (HTA-E; Casiglia \& Tikhonoff, 2018; Soboka, Gudina \& Tesfaye, 2017). Asimismo, en diversas investigaciones, y con el objetivo de aumentar la adherencia al tratamiento médico en estos pacientes, el abordaje de sus factores psicológicos comórbidos han cobrado, de igual forma, un creciente interés (Amico, Mugavero,
Krousel-Wood, Bosworth \& Merlin, 2017; Dyussenova et al., 2018; Sanz et al., 2010; Yu et al., 2015).

En este sentido, entre las dimensiones del afecto negativo estudiadas en la literatura destacan la depresión, la ansiedad y la ira, y, en términos generales, se hipotetiza que las personas que presentan estados afectivos displacenteros con más frecuencia e intensidad son más proclives a padecer HTA y patologías asociadas - como las cardiovasculares-, o incluso mantener estados crónicos de presión arterial (Redina \& Markel, 2018). No obstante, si bien en una serie de estudios se ha encontrado evidencia a favor de dicha hipótesis (véase, por ejemplo, la revisión de Kaplan \& Nunes, 2003, o el metaanálisis de Rutledge \& Hogan, 2002), los resultados no son consistentes. En algunas investigaciones más actuales se ha encontrado, por ejemplo, para la depresión, más evidencia en contra (Bajkó et al., 2012; Uceda, Sanz-Fernández, Espinosa-López \& GarcíaVera, 2013; Yan et al., 2004) que a favor (Meyer, Armenian, Eaton \& Ford, 2004) de dicha hipótesis; mientras que para la ansiedad hay más datos favorables (Bajkó et al., 2012; Uceda et al., 2013; Yu et al., 2015) que desfavorables (Yan et al., 2004) respecto a ella; al igual que en el estudio de la ira, donde han sido más los resultados en favor (Mushtaq \& Najam, 2014; Sahraian et al., 2015; Yan et al., 2004) que en contra (Uceda et al., 2013).

De igual forma, las causas que explican la existencia de resultados dispares aún son fuente de controversia, dado que no es posible establecer si las diferencias se deben a problemas metodológicos o bien a una débil asociación entre las variables (García-Vera, Sanz, Espinosa, Fortún \& Magán, 2010; Sorsdahl et al., 2016; Tikhonoff et al., 2014).

Específicamente, con respecto a los aspectos metodológicos, los antecedentes son muy dispares en cuanto a 
los instrumentos, las poblaciones, los rangos de edades, el porcentaje de hombres y mujeres, la forma de calcular los puntajes de las variables consideradas - p. ej., escalas unidimensionales vs. multidimensionales-, la medición simultánea de las variables afectivas, y el tipo de diseño aplicado — p. ej., longitudinal vs. transversal/caso-control一, razones por las cuales es preciso hacer algunas distinciones metodológicas.

Una primera distinción de relevancia a la hora de comprender la asociación entre las variables es la identificación del diseño utilizado en las investigaciones previas, aspecto que resulta importante porque, mientras los estudios longitudinales posibilitan indagar la capacidad de predicción de las variables afectivas sobre la HTA, los estudios transversales o de caso-control no permiten sacar conclusiones definitivas respecto a la causalidad implicada entre las variables involucradas (Woodward, 2014) y más probablemente evidencian la experiencia afectiva de las personas luego de ser diagnosticados con una enfermedad crónica como la HTA (Yu et al., 2015).

Asimismo, otro aspecto relevante por considerar es si la medida de las variables afectivas se realiza de manera simultánea o separada, aspecto de suma importancia para comprender la asociación entre el afecto negativo y la HTA, ya que el nivel de comunalidad existente entre depresión, ansiedad e ira puede proporcionar una explicación sobre las inconsistencias observadas en algunos estudios (GarcíaVera et al., 2010). De esta forma, si un estudio reporta una asociación positiva entre una variable afectiva y la HTA, esta puede deberse a la acción específica de ella variable sobre la HTA o, simplemente, estar reflejando una correlación con otra variable afectiva que sí se relaciona con la HTA (Suls, 2017). Otra posibilidad es que sea el efecto conjunto - como la comunalidad — más que el efecto individual — como la especificidad - de las variables afectivas lo que permite explicar el surgimiento y mantenimiento de la HTA (Suls \& Bunde, 2005; véase, además, Woodward, 2014, para una explicación mediante diagramas de Venn de todas estas posibilidades).

Hasta la fecha, solo se han podido encontrar dos antecedentes que indagan de manera conjunta estas tres variables afectivas - depresión, ansiedad e ira - en personas hipertensas, en comparación con un grupo control (García-Vera et al., 2010; Uceda et al., 2013), y sus resultados han sido dispares. En la primera, de García-Vera et al. (2010), se encontraron mayores niveles de rasgo de ansiedad y rasgo depresivo en el grupo de HTA, pero no diferencias respecto al rasgo de ira; mientras que, en la segunda, de Uceda et al. (2013), se encontró que las personas con HTA solo se diferenciaban por sus niveles de ansiedad. Según esto, aquí tampoco es posible determinar con certeza las relaciones propuestas.

Dada la limitación de estudios y la inconsistencia en los mismos, la presente investigación tuvo como principal objetivo analizar de manera conjunta medidas de ansiedad, depresión e ira en personas con HTA, es decir, indagar la afectividad negativa comórbida (Rafanelli, Offidani, Gostoli \& Roncuzzi, 2012; Soboka et al., 2017; Tel, 2013) en dichos pacientes. Para superar las principales limitaciones metodológicas identificadas en los antecedentes (GarcíaVera et al., 2010; Uceda et al., 2013), en este trabajo se propuso (a) usar la versión completa del BDI-II para la medición de la depresión, porque permite distinguir entre factores cognitivos, afectivos, y somáticos; (b) implementar el STAXI-2, porque contempla las dos dimensiones principales identificadas para la medición del rasgo de ira - es decir, el temperamento de ira y la reacción-; y (c) aplicar el Inventario de Ansiedad (STAI) de GuillenRiquelme y Buela-Casal (2015), porque permite medir el rasgo de ansiedad en un periodo más amplio al indagar los síntomas ansiosos.

Sumado a lo anterior, y con el fin de lograr mayor precisión en la delimitación de los componentes comunes asociados al HTA, se efectuó un análisis que contempló cada síntoma afectivo específico - la irritabilidad, por ejemplo- más allá de la dimensión general — como la depresión - . De esta forma, mediante la comparación de grupos se buscó analizar la sintomatología emocionalafectiva más característica en personas con HTA, ya que identificar estos síntomas afectivos permite esclarecer dichas asociaciones e identificar con más precisión los síntomas implicados en el mantenimiento de la HTA (Tully, Peters, Pérès, Anstey \& Tzourio, 2018), aspecto que hasta el momento no ha sido indagado.

Resta por señalar que investigaciones precedentes han hecho hincapié en la necesidad de replicar estos estudios en regiones como Latinoamérica y el Caribe (Camacho, Echeverría, Barros, Maiz \& Rigotti, 2018), tema que va en línea con los recientes señalamientos de la Organización Panamericana de la Salud (Khon et al., 2018), en donde se indica la importancia de replicar en las regiones de Centro y Sudamérica los estudios de los países más desarrollados. La consideración de otro tipo de poblaciones es relevante dado que, para el caso de la relación entre la HTA y la afectividad negativa, permite distinguir aspectos de dicha asociación que serían invariantes de aquellos que estarían sujetos a la variabilidad del contexto y la cultura (Rad, Martingano \& Ginges, 2018). Por último, cabe resaltar que en el presente estudio se analiza por primera vez la asociación entre la HTA y la afectividad negativa en población de República Dominicana. 
196

Método

\section{Participantes}

Participó un total de 100 adultos seleccionadas por medio de un muestreo no probabilístico e intencional. Los detalles en cuanto a la forma en que se procedió para seleccionar a los participantes se describen en el apartado de procedimiento. Para definir la categoría "adultos" se siguieron los criterios internacionales (Ruiz, 2005; Vos et al., 2017), según los cuales se pueden considerar como adultos a las personas en el rango etario de 20 a 60 años. En particular, se contrastaron dos grupos, uno conformado por personas que padecían hipertensión arterial, el Grupo $\operatorname{HTA}(n=50)$, y otro que sirvió de control, equiparado en edad y sexo, el Grupo Control $(n=50)$. En cuanto a la variable sexo, en ambos grupos se mantuvo la misma proporción, con un $64 \%$ de mujeres y un $36 \%$ de varones. Respecto a la edad, el rango estuvo entre 23 y 60 años en ambos grupos $\left(M_{\text {Grupo HTA }}=44.72, D E_{\text {Grupo HTA }}=10.67 ; M_{\text {Grupo Control }}=44.26\right.$, $\left.D E_{\text {Grupo Control }}=10.13 ; \Delta \mathrm{M}_{\text {Edad }}=.46, t=.22, g l=98, p=.83\right)$.

\section{Instrumentos}

Inventario de Ansiedad Estado/Rasgo (STAI).

Este cuestionario, en su versión validada en la población Dominicana por García-Batista et al. (2017), evalúa la ansiedad estado y la ansiedad rasgo mediante 40 reactivos -20 para cada una- con una escala de respuesta tipo Likert de cuatro alternativas. En dicha población, las puntuaciones presentaron propiedades psicométricas adecuadas ( $\alpha$ entre .83 y .86), y relaciones significativas con la depresión. En el presente trabajo se consideró solamente la medida de ansiedad rasgo (STAI-R), la cual presentó una consistencia interna óptima $(\alpha=.83)$.

Inventario de Expresión de Ira Rasgo-Estado (STAXI-2).

El STAXI-2, también en su versión validada por GarcíaBatista et al. (2018a) en República Dominicana, ofrece una evaluación que diferencia los distintos elementos de la ira - la experiencia, la expresión y el control—, así como sus facetas tanto de estado como de rasgo, por medio de 49 reactivos y una escala de respuesta tipo Likert de cuatro alternativas. Los resultados en el estudio de García-Bautista et al. (2018) dan cuenta de una consistencia interna aceptable y óptima ( $\alpha$ entre .75 y .86) y diferencias significativas entre población general y clínica; al igual que en el presente estudio - en el que se consideró solo la medida de rasgo de ira-, donde tanto la dimensión de temperamento $(\alpha=.84)$ como la de reacción $(\alpha=.82)$ presentaron una consistencia interna óptima.

Inventario de depresión de Beck II (BDI-II).

Este instrumento, originalmente diseñado por Beck, Steer y Brown (1996), es un inventario de autoinforme basado en los síntomas descritos por el Manual Diagnóstico y Estadístico de los Desórdenes Mentales (DSM-IV; American Psychiatric Association, 2000), que permite la medición de la severidad depresiva. La versión del inventario utilizada en el presente estudio - validada para a la población dominicana por García-Batista, Guerra-Peña, Cano-Vindel, Herrera-Martínez y Medrano (2018b) — consiste en 21 ítems con cuatro opciones de respuesta en escala Likert de 0 a 3 que evalúan la severidad depresiva de manera general, así como los subcomponentes cognitivos, afectivos y somáticos de la depresión. Específicamente, la validación dominicana permitió constatar la adecuación de un modelo bifactorial $\left(\chi^{2}=541.57\right.$; CFI $=.94 ;$ RMSEA $\left.=.05\right)$, así como valores de consistencia interna óptimos para la dimensión general $(\alpha=.89)$ y aceptables para las subdimensiones específicas (valores $\alpha$ entre .70 y .78). En el presente estudio se obtuvieron valores aceptables para las tres dimensiones consideradas: la cognitiva $(\alpha=.78)$, la somática $(\alpha=.77)$ y la afectiva $(\alpha=.70)$.

\section{Procedimiento}

La investigación sigue un diseño de caso-control (Woodward, 2014). El grupo de personas con hipertensión arterial fue seleccionado en las consultas del Hospital José María Cabral y Báez, localizado en la ciudad de Santiago de Los Caballeros, República Dominicana. Aquí, las personas diagnosticadas con HTA por los doctores especialistas en cardiología eran referidas al equipo de investigadores. En un segundo momento, se acordó un encuentro con este grupo para aplicar las escalas correspondientes; allí se les explicó de forma clara y breve los objetivos de la investigación, asegurando su participación voluntaria y anónima, tras lo cual procedieron a firmar el consentimiento, se tomaron los datos del paciente y se aplicaron los instrumentos.

Posteriormente, se seleccionó una muestra de personas sin problemas de salud, buscando que el grupo control tuviera características similares en cuanto a cantidad de casos, sexo y edad con respecto al grupo de HTA. Una vez que estos accedían, se les explicó también de forma clara y breve los objetivos de la investigación, asegurando su participación voluntaria y anónima. Igualmente, se obtuvo el consentimiento verbal y se aplicaron las pruebas correspondientes. 
La presente investigación contó con la aprobación del Comité Nacional de Bioética (CONABIOS) perteneciente al Ministerio de Salud Pública de Santo Domingo, República Dominicana (protocolo número 028-2014).

\section{Análisis de datos}

La selección del procedimiento estadístico para identificar las diferencias entre los grupos de interés quedó determinada por el cumplimiento o no de los supuestos de normalidad y homocedasticidad, para los cuales se aplicó la Prueba de Normalidad de Kolmogorov-Smirnov y la prueba de Levene para la igualdad de varianzas (Tabachnick \& Fidell, 2007). Como se podrá apreciar en las tablas 1 y 3 , estos supuestos se incumplen en mayor o menor grado, motivo por el cual se decidió aplicar pruebas robustas modernas, sugeridas en casos de apartamiento de los datos a los supuestos de normalidad y homogeneidad de varianza (Wilcox \& Keselman, 2003). En términos generales, este grupo de pruebas denominadas robustas modernas - como la prueba de Yuen, la prueba de Yuen con Boostrap, o el percentil-boostrap basado en el estimador $\mathrm{M}$ - no hacen asunciones respecto a la forma funcional de la distribución de probabilidad (Wilcox, 2012).

En concreto, para identificar las diferencias entre los grupos se calcularon medidas de posición robustas, la media recortada al $10 \%$, así como su desviación estándar correspondiente (Wilcox, 2012); y se aplicó la prueba de Yuen - también basada en una media recortada al 10\%-, dado que ha demostrado ser una de las pruebas robustas modernas más óptimas en caso de violación de los supuestos de normalidad y homocedasticidad, principalmente en términos de control del error tipo 1 y mantenimiento de la potencia de la prueba (Ozdemir, Wilcox \& Yildiztepe,
2012). El procedimiento anterior se mantuvo tanto a nivel general, o dimensional, como a nivel de los síntomas, pero, respecto a los síntomas específicos, las puntuaciones se obtuvieron mediante una escala tipo Likert —es decir, ordinal- En cuanto a este último punto, la literatura sugiere que pueden aplicarse pruebas de tipo inferencial, como las paramétricas o las robustas modernas propuestas en la presente investigación (Norman, 2010).

Adicionalmente, para comprobar la magnitud de las diferencias se calculó el tamaño del efecto mediante la $d$ de Cohen (Lakens, 2013), con una versión robusta de ella, propuesta por Algina, Keselman y Penfield (2005), que se basa en la media recortada. Para los análisis mencionados se utilizaron los softwares estadísticos R (R Core Team, 2016) e IBM SPSS, v. 20.

\section{Resultados}

A continuación se describen los resultados obtenidos, organizados en dos partes principales: primero se presentan los análisis exploratorios iniciales y las diferencias entre grupos, considerando las dimensiones generales o globales de la depresión, la ansiedad y la ira; y segundo, el mismo procedimiento, pero a nivel de los síntomas.

\section{Análisis exploratorio y descriptivo inicial: niveles generales de afectividad negativa}

Antes de verificar si los grupos diferían en los niveles generales de depresión, rasgo de ansiedad e ira, se llevaron a cabo análisis exploratorios y se aplicaron las pruebas de normalidad correspondientes (véase Tabla 1).

Tabla 1.

Análisis exploratorio de los datos a nivel global: asimetría, curtosis, prueba de normalidad y homocedasticidad

\begin{tabular}{|c|c|c|c|c|c|}
\hline & \multicolumn{2}{|c|}{ Descriptivos } & \multicolumn{3}{|c|}{ Prueba de supuestos } \\
\hline & $\begin{array}{c}\text { Con HTA } \\
\text { g1/g2 }\end{array}$ & $\begin{array}{c}\text { Sin HTA } \\
\text { g1/g2 }\end{array}$ & $\begin{array}{c}\text { Con HTA } \\
D\end{array}$ & $\begin{array}{c}\text { Sin HTA } \\
D\end{array}$ & $\begin{array}{l}\text { Estadístico de } \\
\text { Levene }\end{array}$ \\
\hline \multicolumn{6}{|l|}{ Depresión } \\
\hline Cognitivo & $1.13 / 0.41$ & $1.57 / 1.51$ & $.21 * *$ & $.28 * *$ & 2.17 \\
\hline Afectivo & $1.19 /-0.17$ & $2.51 / 6.91$ & $.32 * *$ & $.27 * *$ & $5.00 *$ \\
\hline Somático & $0.82 / 1.15$ & $1.38 / 2.23$ & .11 & $.17 * *$ & 1.58 \\
\hline \multicolumn{6}{|l|}{ Ansiedad } \\
\hline Rasgo & $0.58 /-0.30$ & $0.41 /-0.57$ & $.16^{* *}$ & .12 & 0.54 \\
\hline \multicolumn{6}{|l|}{ Ira rasgo } \\
\hline Temperamento & $1.63 / 2.22$ & $1.02 / 0.18$ & $.22 * *$ & $.22 * *$ & $5.65 *$ \\
\hline Reacción & $0.69 /-0.09$ & $0.90 / 0.71$ & $.15^{* *}$ & $.14^{* *}$ & 0.99 \\
\hline
\end{tabular}

Nota. ${ }^{*} \mathrm{p}<.05,{ }^{*} \mathrm{p}<.01 ; \mathrm{g} 1=$ asimetría; $\mathrm{g} 2=$ curtosis; $D=$ estadístico de la prueba de normalidad de Kolmogorov-Smirnov. 
En términos generales, puede verse el alejamiento de los datos a la normalidad, y, en algunos casos, el incumplimiento del supuesto de igualdad de varianza.

Análisis de diferencias entre grupos en los niveles generales de afectividad negativa: pruebas inferenciales y medidas del tamaño de efecto

A continuación, en la Tabla 2 se muestran las diferencias verificadas entre los grupos contrastados por medio de la aplicación de métodos estadísticos robustos.
Como se puede observar, se evidencian diferencias estadísticamente significativas en favor del grupo de HTA solo en dos de las dimensiones evaluadas, a saber, mayores niveles de depresión — en su dimensión somática- y de rasgo de ira - en su dimensión de temperamento- - En cuanto al tamaño del efecto, la depresión —en su dimensión somática — da cuenta de la diferencia más fuerte evidenciada entre los grupos. Las deferencias constatadas tanto para el caso de la depresión somática como para el temperamento de ira resultan ser de magnitud moderada.

Tabla 2.

Niveles generales de depresión, rasgo de ansiedad e ira entre el grupo con HTA y el grupo control

\begin{tabular}{|c|c|c|c|c|c|}
\hline & \multicolumn{2}{|c|}{ Grupo } & \multirow{3}{*}{$\mathrm{T}_{y}$} & \multirow{3}{*}{$p$} & \multirow{3}{*}{$d$} \\
\hline & Con HTA & Sin HTA & & & \\
\hline & T-M (DE) & T-M (DE) & & & \\
\hline Depresión & & & & & \\
\hline Cognitivo & $2.75(.53)$ & $1.65(.47)$ & 1.17 & .13 & .31 \\
\hline Afectivo & $1.80(.50)$ & $0.90(.24)$ & 1.60 & .11 & .33 \\
\hline Somático & $7.27(.71)$ & $4.62(.53)$ & 2.96 & $<.01$ & .61 \\
\hline Ansiedad & & & & & \\
\hline Rasgo & $14.75(1.30)$ & $12.70(1.15)$ & 1.17 & .24 & .24 \\
\hline Ira rasgo & & & & & \\
\hline Temperamento & $8.15(.46)$ & $6.77(.32)$ & 2.40 & .01 & .50 \\
\hline Reacción & $11.07(.58)$ & $9.75(.44)$ & 1.80 & .07 & .37 \\
\hline
\end{tabular}

Nota. T-M = trimmed mean (media recortada al $10 \%$ ); $\mathrm{T}_{y}=$ Yuen's test; $\mathrm{DE}=$ desviación estándar. En negrilla se resaltan los puntajes que presentaron una diferencia estadísticamente significativa.

Tabla 3.

Análisis exploratorio de los datos a nivel de la sintomatología: asimetría, curtosis y prueba de normalidad

\begin{tabular}{|c|c|c|c|c|c|}
\hline & \multicolumn{2}{|c|}{ Descriptivos } & \multicolumn{2}{|c|}{ Prueba de supuestos } & \multirow[b]{2}{*}{$\begin{array}{c}\text { Estadístico de } \\
\text { Levene }\end{array}$} \\
\hline & $\begin{array}{c}\text { Con HTA } \\
\text { g1/g2 } \\
\end{array}$ & $\begin{array}{c}\text { Sin HTA } \\
\mathrm{g} 1 / \mathrm{g} 2 \\
\end{array}$ & $\begin{array}{c}\text { Con HTA } \\
D \\
\end{array}$ & $\begin{array}{c}\text { Sin HTA } \\
D \\
\end{array}$ & \\
\hline \multicolumn{6}{|l|}{ Depresión: Somático } \\
\hline 15. Pérdida de energía. & $0.62 / 0.18$ & $1.16 / 1.98$ & $.27 * *$ & $.30 * *$ & 0.03 \\
\hline 17. Irritabilidad. & $1.64 / 2.72$ & $2.54 / 6.06$ & $.32 * *$ & $.46^{* *}$ & 1.86 \\
\hline 19. Dificultad en la concentración. & $0.67 /-0.53$ & $1.23 / 1.65$ & $.25 * *$ & $.32 * *$ & 2.80 \\
\hline 20. Fatiga. & $0.78 /-0.36$ & $1.41 / 2.33$ & $.27 * *$ & $.26 * *$ & 0.96 \\
\hline 10. Llanto. & $1.14 / 0.16$ & $1.14 /-0.35$ & $.36^{* *}$ & $.40 * *$ & $4.11 *$ \\
\hline 11. Agitación. & $1.37 / 0.77$ & $2.34 / 5.38$ & $.30 * *$ & $.41 * *$ & 3.68 \\
\hline 16. Cambios en los hábitos de sueño. & $0.21 /-.73$ & $0.76 /-0.01$ & $.23 * *$ & $.26^{* *}$ & 0.43 \\
\hline 18. Cambios de apetito. & $0.29 /-1.02$ & $1.11 / 0.64$ & $.21 * *$ & $.28 * *$ & 0.49 \\
\hline 21. Pérdida de interés sexual. & $1.38 / 1.06$ & $2.19 / 4.11$ & $.30 * *$ & $.41 * *$ & 1.14 \\
\hline \multicolumn{6}{|l|}{ Ira: Temperamento } \\
\hline 16. Me caliento rápidamente. & $0.98 / 0.21$ & $1.50 / 2.96$ & $.32 * *$ & $.30 * *$ & 0.09 \\
\hline 17. Tengo un carácter irritable. & $1.28 / 1.04$ & $0.60 /-1.70$ & $.28 * *$ & $.41 * *$ & $10.92 * *$ \\
\hline 18. Soy una persona exaltable. & $1.45 / 1.37$ & $1.03 /-0.02$ & $.35 * *$ & $.41 * *$ & $10.26^{* *}$ \\
\hline 20. Tiendo a perder los estribos. & $1.69 / 3.40$ & $2.48 / 8.26$ & $.34 * *$ & $.43^{* *}$ & $4.15^{*}$ \\
\hline 23. Me enojo con facilidad. & $1.04 / 0.13$ & $1.68 / 2.72$ & $.28 * *$ & $.39 * *$ & 1.96 \\
\hline
\end{tabular}

Nota. $* \mathrm{p}<.05, * * \mathrm{p}<.01 . \mathrm{g} 1=$ asimetría; $\mathrm{g} 2=$ curtosis. $D=$ estadístico de la prueba de normalidad de Kolmogorov-Smirnov. 
Análisis exploratorio y descriptivo inicial: nivel sintomático

Finalmente, se analizaron las diferencias a nivel de los síntomas, contemplando la depresión en cuanto al factor somático y el temperamento respecto de la ira, dado que, a nivel global, fueron las únicas que dieron cuenta de diferencias estadísticamente significativas entre los grupos. Al igual que al analizar las variables de manera dimensional, previamente se llevaron a cabo análisis exploratorios y se aplicaron las pruebas de normalidad y homocedasticidad respectivas (véase Tabla 3 ).

A nivel de los síntomas, puede comprobarse también un alejamiento de los datos a la normalidad, y en algunos casos el incumplimiento del supuesto de igualdad de varianza.

Análisis de diferencias entre grupos en los niveles sintomáticos de afectividad negativa: pruebas inferenciales y medidas del tamaño de efecto

A continuación, en la Tabla 4 se pueden apreciar las diferencias identificadas a nivel sintomático entre los grupos contrastados por medio de la aplicación de métodos estadísticos robustos.

Como se puede observar, los síntomas depresivos somáticos más característicos del grupo con HTA corresponden a la irritabilidad $(d=.54)$ y la pérdida de energía $(d=.50)$, seguidos por los cambios de apetito $(d=.44)$ y la dificultad en la concentración $(d=.42)$; mientras que para el temperamento de ira fueron el enojarse con facilidad $(d=.50)$ y calentarse rápidamente $(d=.49)$. En todos estos síntomas las diferencias evidenciadas presentan un tamaño de efecto moderado.

\section{Discusión}

En la presente investigación se propuso como principal objetivo analizar en conjunto medidas de ansiedad, depresión e ira en personas con HTA, y, de manera complementaria, se planteó identificar la sintomatología afectiva más característica en estos pacientes.

Respecto al primer objetivo, si bien en general en todas las variables se observan mayores niveles en el grupo de HTA, solo en el factor somático de la depresión y en el temperamento de ira se observaron diferencias estadísticamente significativas - y de moderada magnitud — en favor de las personas con HTA. En cuanto al segundo objetivo, los síntomas más característicos de las personas con HTA refieren a la irritabilidad y la pérdida de energía para el caso de los síntomas depresivos somáticos; mientras que para el temperamento de ira la sintomatología más característica refiere a enojarse con facilidad y calentarse rápidamente.

A la fecha, solo se han detectado dos antecedentes con objetivos similares, y, en general, los resultados de la presente investigación difieren de los hallazgos previos, exceptuando el caso de la depresión, pues se encontraron datos similares a los obtenidos por García-Vera et al., (2010).

Tabla 4.

Niveles de sintomatología afectiva: comparación entre el grupo con HTA y el grupo control

\begin{tabular}{|c|c|c|c|c|c|}
\hline & \multicolumn{2}{|c|}{ Grupos } & \multirow{3}{*}{$\mathrm{T}_{y}$} & \multirow{3}{*}{$p$} & \multirow{3}{*}{$d$} \\
\hline & Con HTA & Sin HTA & & & \\
\hline & $\mathrm{T}-\mathrm{M}(D E)$ & $\mathrm{T}-\mathrm{M}(D E)$ & & & \\
\hline \multicolumn{6}{|l|}{ Depresión: Somático } \\
\hline 15. Pérdida de energía. & $0.87(.12)$ & $0.50(.08)$ & 2.43 & .01 & .50 \\
\hline 17. Irritabilidad. & $0.42(.08)$ & $0.12(.07)$ & 2.62 & .01 & .54 \\
\hline 19. Dificultad en la concentración. & $0.80(.14)$ & $0.45(.08)$ & 2.05 & .04 & .42 \\
\hline 20. Fatiga. & $0.92(.17)$ & $0.55(.08)$ & 1.87 & .06 & .38 \\
\hline 10. Llanto. & $0.50(.14)$ & $0.55(.20)$ & 0.20 & .84 & .04 \\
\hline 11. Agitación. & $0.55(.18)$ & $0.22(.08)$ & 1.63 & .10 & .33 \\
\hline 16. Cambios en los hábitos de sueño. & $1.00(.13)$ & $0.65(.12)$ & 1.87 & .06 & .38 \\
\hline 18. Cambios de apetito. & $1.02(.14)$ & $0.60(.13)$ & 2.13 & .03 & .44 \\
\hline 21. Pérdida de interés sexual. & $0.50(.12)$ & $0.22(.08)$ & 1.80 & .07 & .37 \\
\hline \multicolumn{6}{|l|}{ Ira: Temperamento } \\
\hline 16. Me caliento rápidamente. & $1.87(.17)$ & $1.47(.08)$ & 2.06 & .04 & .42 \\
\hline 17. Tengo un carácter irritable. & $1.55(.12)$ & $1.32(.08)$ & 1.46 & .14 & .30 \\
\hline 18. Soy una persona exaltable. & $1.42(.12)$ & $1.30(.08)$ & 0.80 & .42 & .16 \\
\hline 20. Tiendo a perder estribos. & $1.40(.08)$ & $1.20(.07)$ & 1.69 & .09 & .35 \\
\hline 23. Me enojo con facilidad. & $1.77(.17)$ & $1.30(.08)$ & 2.40 & .01 & .50 \\
\hline
\end{tabular}

Nota. $\mathrm{T}-\mathrm{M}=$ trimmed mean (media recortada al $10 \%$ ); $\mathrm{T}_{v}=$ Yuen's test $\mathrm{DE}=$ desviación estándar. En negrilla se resaltan las puntuaciones que presentaron una diferencia estadísticamente significativa. 
Estos resultados opuestos pueden explicarse, en parte, debido a las diferencias metodológicas entre los estudios.

Específicamente, con respecto a la medición de la depresión, en el presente estudio se utilizó la versión completa del BDI-II, que consta de 21 ítems y posibilita la distinción de tres dimensiones específicas, mientras que en el estudio de Uceda et al. (2013) se aplicó la versión corta del Inventario de Depresión de Beck (BDI-II-SV), elaborada por Sanz, García-Vera, Fortún y Espinosa (2005), de la cual solo se tomó el puntaje total de dicha escala. Esta forma de proceder hace más difícil la detección de los rasgos depresivos somáticos identificados por el presente estudio (García-Batista et al., 2018b).

En cuanto a la ansiedad, también se presentaron diferencias con respecto al instrumento aplicado, ya que, aunque en el estudio de Uceda et al. (2013) se aplicó el Inventario de Ansiedad de Beck (BAI), instrumento que tiene ciertas semejanzas psicométricas con el STAI-R, este presenta diferencias notables en lo que respecta a los constructos que pretenden medir, el contenido de sus ítems - como los síntomas de ansiedad y de depresión que evalúan dichos reactivos - y sus capacidades para discriminar entre ansiedad y depresión (Sanz, 2014).

Más precisamente, como indica el relevamiento de Sanz (2014), el STAI-R es más adecuado que el BAI para evaluar el trastorno de ansiedad generalizada, ya que cubre más del $50 \%$ de síntomas de este trastorno, mientras que el BAI es más adecuado para la evaluación de crisis de angustia, pues cubre más del $50 \%$ de síntomas de esta. Incluso, una limitación importante del STAI-R con respecto al BAI concierne a la validez de contenido, dado que el $65 \%$ de los ítems del STAI-R miden también síntomas del episodio depresivo mayor. Sintetizando, por un lado, en ambos estudios se midieron síntomas de ansiedad diferentes, lo cual indica que en el presente estudio no se encuentra evidencia de que los síntomas del TAG sean característicos de las personas con HTA; y, por otro, en el caso del presente trabajo, la falta de una distinción clara entre los grupos comparados tal vez pueda explicarse también por el solapamiento de síntomas depresivo-ansiosos en las puntuaciones del STAI-R (Sanz, 2014). Lo anterior permitiría explicar, además, que los resultados no sean consistentes entre los estudios que aplican dicho instrumento (García-Vera et al., 2010). Por último, las discordancias también podrían radicar en las diferencias en los lapsos que consideran ambos instrumentos para medir los síntomas de ansiedad. Futuras investigaciones deberán dar mayor precisión sobre estas consideraciones.

Con respecto al rasgo de ira, tanto García-Vera et al., (2010) como Uceda et al., (2013) aplicaron, al igual que el presente estudio, el STAXI-2, sin embargo, en ellos se toma un puntaje total, mientras que en el presente estudio se toma la definición original de Spielberger (1991) con respecto a las dos dimensiones del rasgo de ira: (a) el temperamento irritable, que contempla la predisposición general a experimentar y expresar enojo sin una provocación específica; y (b) la reacción de ira, que contempla la predisposición a expresar enojo a partir de una provocación específica. Al respecto, es importante tener en cuenta que en los estudios psicométricos del STAXI-2 esta estructura bifactorial es la que presenta mayor evidencia favorable (García-Batista et al., 2018a), y que la medición multidimensional de la ira ha sido planteada como condición necesaria para llegar a conclusiones y resultados más certeros respecto a su vinculación con la HTA (Siegel, 1986). Por lo tanto, como ocurrió con la medición de la depresión, se considera que el haber contemplado las subdimensiones concernientes al rasgo de ira haría más probable la verificación de diferencias entre los grupos.

Por otra parte, una novedad importante de la presente investigación radica en la identificación de rasgos de sintomatología depresiva y de ira características de personas que padecen HTA. Al respecto, los resultados arrojan que estas personas presentan en mayor medida síntomas de irritabilidad y pérdida de energía — síntomas depresivo somáticos-, además de que se enojan con facilidad o rápidamente síntomas de temperamento de ira-; si bien no existen antecedentes sobre los síntomas afectivos específicos en personas con HTA, sí hay evidencia de que suelen padecer depresión, lo cual produce un detrimento de su calidad de vida (Rafanelli et al., 2012).

Asimismo, se ha encontrado que los síntomas depresivos correlacionan tanto con la duración del diagnóstico - es decir, el tiempo de padecimiento de la enfermedad - como con la experimentación de ira en las personas hipertensas (Tel, 2013); y, en correspondencia, Rafanelli et al. (2012) han evidenciado que las personas con HTA padecen de depresión y estado de ánimo irritable. Esta comorbilidad afectiva observada se explicaría en función de que las personas que padecen enfermedades médicas crónicas como la hipertensión presentan dificultades en el ajuste o adaptación de sus aspiraciones, estilo de vida y empleo (Soboka et al., 2017). Así, la presencia de la enfermedad podría dar lugar al surgimiento de esta sintomatología, la cual podría mantenerse en el nivel subclínico y, no obstante, repercutir en su salud física (Rafanelli et al., 2012).

En suma, la asociación que se propone entre los afectos y la HTA debe entenderse de manera recursiva y no unidireccional, similar a lo propuesto recientemente para las enfermedades cardiovasculares (Davidson, Alcántara \& Miller, 2018; Zhang, Chen \& Ma, 2018). De este modo, la 
afectividad negativa no solo debe ser vista como un factor de riesgo para padecer un trastorno como la HTA, sino que, además, algunos afectos negativos pueden presentarse una vez se diagnostique la HTA, lo que empeoraría el cuadro posterior mediante mecanismos biológicos o conductuales (véase, por ejemplo, Jaén-Águila et al., 2014; Moxotó \& Malagris, 2015; Özpelit et al., 2015; o Yu et al., 2015).

Finalmente, es importante mencionar algunas de las limitaciones del presente trabajo. Por una parte, el diseño de tipo caso-control no permite sacar conclusiones definitivas con respecto a la causalidad o relación temporal de las variables involucradas (Woodward, 2014; Yu et al., 2015), así, el paso de diseños transaccionales a longitudinales permitirá en futuras investigaciones hacer inferencias más rigurosas sobre las relaciones causales implicadas (Cole \& Maxwell, 2003). Por otra parte, los instrumentos y métodos aplicados para medir las variables presentan otra limitación, ya que se utilizaron medidas de autoinforme, algunas de ellas discutibles en cuanto a su validez de contenido o discriminante, como sucede con el STAI. Con respecto a esto último, metaanálisis recientes han evidenciado que los estudios que han usado medidas de autoinforme muestran resultados menos consistentes que los estudios que usan entrevistas estructuradas de diagnóstico (Yu et al., 2015).

Otra de las limitaciones es que para el caso de la variable HTA solo se consideró la presencia o ausencia de la misma — es decir, una medida clínica—, por lo que resulta relevante que futuros estudios también contemplen medidas complementarias, como la presión arterial automedida o ambulatoria (véase, por ejemplo, Bajkó et al., 2012; Jaén-Águila et al., 2014; o Uceda et al., 2013). Aunque es probable que la mayoría de la muestra con HTA tomada en el presente estudio haya tenido una hipertensión primaria o esencial, la presencia de hipertensión secundaria - es decir, causada por otra condición médica establecida-, o de la HTA enmascarada o clínica — que no refleja una patología crónica, sino una presión arterial alta momentánea o circunstancial (Uceda et al., 2013) - no fueron descartadas en estos participantes. Si bien el objetivo central de este trabajo no fue indagar las causas de la HTA, sino las consecuencias afectivas de padecer de dicha patología, es preciso tener una clara delimitación de la muestra, por lo cual, en concordancia con García-Vera et al., (2010), en futuros estudios se debe poder identificar claramente el tipo de hipertensión que padecen los participantes. Otro dato relevante no considerado aquí fue el tiempo transcurrido desde que se diagnosticó la HTA.

Por último, es importante que se incluyan otras medidas sociodemográficas de control que han mostrado asociación con indicadores de afectividad negativa en personas con
HTA, como pueden ser el nivel educativo y el estado civil (Soboka et al., 2017).

Más allá de las anteriores consideraciones, el presente estudio permite empezar a recabar evidencia en poblaciones en donde hasta el momento se carecía de investigaciones previas. Además, resulta un aporte a la literatura internacional en función de que se ha dejado mayor constancia de la sintomatología de afectividad negativa específica característica de personas con HTA. Esto último no solo tiene implicancias a nivel teórico, sino que, además, permite pensar en tratamientos psicoterapéuticos basados en la evidencia teniendo en cuenta a las personas que padecen de esta condición crónica (Fu et al., 2015); e, incluso, el abordaje de estos factores afectivos comórbidos es relevante para aumentar la adherencia al tratamiento médico en este tipo de pacientes (Amico et al., 2017; Dyussenova et al., 2018; Sanz et al., 2010; Yu et al., 2015).

En conclusión, los datos proporcionados por el presente trabajo permiten afirmar la importancia hacer énfasis en ciertos rasgos de afectividad negativa en personas que ya padecen de HTA, concretamente en los síntomas depresivos somáticos — con especial hincapié en la irritabilidad y la pérdida de energía - y de temperamento de ira - con énfasis en la tendencia o facilidad de presentar respuestas de enojo-, dado que estos síntomas podrían favorecer el mantenimiento de la presión arterial elevada en estos sujetos.

\section{Financiamiento}

Esta investigación contó con el apoyo del Fondo Nacional de Innovación y Desarrollo Científico y Tecnológico (FONDOCYT) de la República Dominicana, por tal motivo, les agradecemos. No existen conflictos de interés con el organismo de financiamiento y/o interferir con la adherencia al tratamiento médico.

\section{Referencias}

Algina, J., Keselman, H. J., \& Penfield, R. D. (2005). An alternative to Cohen's standardized mean difference effect size: a robust parameter and confidence interval in the two independent groups case. Psychological Methods, 10(3), 317328. doi: 10.1037/1082-989x.10.3.317

American Psychiatric Association. (2000). Diagnostic and statistical manual of mental disorders (4. ${ }^{\mathrm{a}}$ ed.). Washington, D. C.: American Psychiatric Association.

Amico, K. R., Mugavero, M., Krousel-Wood, M. A., Bosworth, H. B., \& Merlin, J. S. (2017). Advantages to Using SocialBehavioral Models of Medication Adherence in Research and Practice. Journal of General Internal Medicine, 33(2), 207-215. doi:10.1007/s11606-017-4197-5 
Bajkó, Z., Szekeres, C.-C., Kovács, K. R., Csapó, K., Molnár, S., Soltész, P., ... Csiba, L. (2012). Anxiety, depression and autonomic nervous system dysfunction in hypertension. Journal of the Neurological Sciences, 317, 112-116. doi: 10.1016/j.jns.2012.02.014

Beck, A., Steer, R., \& Brown, G. (1996). Beck Depression Inventory. San Antonio, EE. UU.: Psychological Corporation.

Camacho, J., Echeverría, G., Barros, J., Maiz, A., \& Rigotti, A. (2018). Depression and Stress are Highly Associated with Hypertension, Diabetes, and Cardiovascular Disease in Chilean Population. Atherosclerosis Supplements, 32, 74. doi: 10.1016/j.atherosclerosissup.2018.04. $\dot{A}$

Casiglia, E., \& Tikhonoff, V. (2018). Essential hypertension: the specialist as part of therapeutic intervention. Hypertension Research, 41(5), 323-325. doi:10.1038/s41440-018-0031-7

Cole, D. A., \& Maxwell, S. E. (2003). Testing Mediational Models With Longitudinal Data: Questions and Tips in the Use of Structural Equation Modeling. Journal of Abnormal Psychology, 112(4), 558-577. doi: 10.1037/0021-843x.112.4.558

Davidson, K. W., Alcántara, C., \& Miller, G. E. (2018). Selected psychological comorbidities in coronary heart disease: Challenges and grand opportunities. American Psychologist, 73(8), 1019-1030. doi: 10.1037/amp0000239

Dyussenova, L., Pivina, L., Semenova, Y., Bjørklund, G., Glushkova, N., Chirumbolo, S., \& Belikhina, T. (2018). Associations between depression, anxiety and medication adherence among patients with arterial hypertension: Comparison between persons exposed and non-exposed to radiation from the Semipalatinsk Nuclear Test Site. Journal of Environmental Radioactivity, 195, 33-39. doi: 10.1016/j. jenvrad.2018.09.016

Fu, W., Ma, L., Zhao, X., Li, Y., Zhu, H., Yang, W., ... Liu, H. (2015). Antidepressant medication can improve hypertension in elderly patients with depression. Journal of Clinical Neuroscience. doi:10.1016/j.jocn.2015.03.067

García-Batista, Z. E., Guerra-Peña, K., Cano-Vindel, A., Herrera-Martínez, S. X., \& Medrano, L. A. (2018b). Validity and reliability of the Beck Depression Inventory (BDI-II) in general and hospital population of Dominican Republic. PLOS ONE, 13(6), e0199750. doi: 10.1371/jour nal.pone. 0199750

García-Batista, Z. E., Guerra-Peña, K., Cano-Vindel, A., Herrera-Martínez, S. X., Flores-Kanter, P. E., \& Medrano, L. A. (2017). Propiedades psicométricas del Inventario de ansiedad estado-rasgo en población general y hospitalaria de República Dominicana. Ansiedad y Estrés, 23(2-3), 5358. doi: 10.1016/j.anyes.2017.09.004

García-Batista, Z. E., Guerra-Peña, K., Cano-Vindel, A., Herrera-Martínez, S. X., Flores-Kanter, P. E., \& Medrano, L. A. (2018a). Evidencias de validez y fiabilidad de las Puntuaciones del STAXI-2 para población general y hospitalaria: Estudio con una muestra de adultos de República
Dominicana. Suma Psicológica, 25(1), 21-29. doi: 10.14349/sumapsi.2018.v25.n1.3

García-Vera, M. P., Sanz, J., Espinosa, R., Fortún, M., \& Magán, I. (2010). Differences in emotional personality traits and stress between sustained hypertension and normotension. Hypertension Research, 33(3), 203-208. doi: 10.1038/hr.2009.210

Guillen-Riquelme, A., \& Buela-Casal, G. (2015). Estructura factorial del cuestionario de Ansiedad Estado-Rasgo (STAI) para pacientes diagnosticados con depresión. Salud Mental, 38(4), 293-298. doi: 10.17711/sm.0185-3325.2015.040

Jaén-Águila, F., Mediavilla-García, J. D., Navarrete-Navarrete, N., Ramos-Cortés, J. L., Fernández-Torres, C., \& JiménezAlonso, J. (2014). Ansiedad, depresión y su implicación en la hipertensión arterial resistente. Hipertensión y Riesgo Vascular, 31(1), 7-13. doi: 10.1016/j.hipert.2013.08.001

Kaplan, M. S., \& Nunes, A. (2003). The psychosociai determinants of hypertension. Nutrition, Metabolism and Cardiovascular Diseases, 13(1), 52-59. doi: 10.1016/ S0939-4753(03)80168-0

Kohn, R., Ali, A. A., Puac-Polanco, V., Figueroa, C., LópezSoto, V., Morgan, K., ... Vicente, B. (2018). Mental health in the Americas: an overview of the treatment gap. Revista Panamericana de Salud Pública, 42, e165. doi: 10.26633/ rpsp.2018.165

Lakens, D. (2013). Calculating and reporting effect sizes to facilitate cumulative science: a practical primer for t-tests and ANOVAs. Frontiers in Psychology, 4, 863. doi: 10.3389/ fpsyg.2013.00863

Meyer, C. M., Armenian, H. K., Eaton, W. W., \& Ford, D. E. (2004). Incident hypertension associated with depression in the Baltimore Epidemiologic Catchment area follow-up study. Journal of Affective Disorders, 83(2-3), 127-133. doi: 10.1016/j.jad.2004.06.004

Moxotó, G. A., \& Malagris, L. E. (2015). Raiva, stress emocional e hipertensão: Um estudo comparativo. Psicologia: Teoria e Pesquisa, 31(2), 221-227. doi: 10.1590/0102-37722015021189221227

Mushtaq, M., \& Najam, N. (2014). Anger as a psychological risk factor of hypertension. Pakistan Journal of Psychological Research, 29(1), 21-37. Recuperado de http://www.pjprnip. edu.pk/pjpr/index.php/pjpr/article/view/312

Norman, G. (2010). Likert scales, levels of measurement and the "laws" of statistics. Advances in Health Sciences Education, 15(5), 625-632. doi: 10.1007/s10459-010-9222-y

Organización Mundial de la Salud. (2000). Comparación transnacional de la prevalencia de los trastornos mentales y los factores con ellos correlacionados. Boletín de la Organización Mundial de la Salud: recopilación de artículos 2000, 3, 92-105. Recuperado de https://apps.who. int/iris/handle/10665/58007

Organización Mundial de la Salud. (2013). A Global Brief on Hypertension. Suiza: WHO Press. 
Ozdemir, A. F., Wilcox, R. R., \& Yildiztepe, E. (2012). Comparing Measures of Location: Some Small-Sample Results When Distributions Differ in Skewness and Kurtosis Under Heterogeneity of Variances. Communications in Statistics-Simulation and Computation, 42(2), 407-424. doi: 10.1080/03610918.2011.636163

Özpelit, M. E., Özpelit, E., Doğan, N. B., Pekel, N., Ozyurtlu, F., Yilmaz, A., ... Ercan, E. (2015). Impact of anxiety level on circadian rhythm of blood pressure in hypertensive patients. International Journal of Clinical and Experimental Medicine, 8(9), 16252-16258. Recuperado de http://www. ijcem.com/V8_No9.html

Ploth, D. W., Mbwambo, J. K., Fonner, V. A., Horowitz, B., Zager, P., Schrader, R., ... Sweat, M. D. (2018). Prevalence of CKD, Diabetes, and Hypertension in Rural Tanzania. Kidney International Reports, 3(4), 905-915. doi: 10.1016/j. ekir.2018.04.006

R Core Team. (2016). R: A Language and Environment for Statistical Computing. Vienna, Austria: R Foundation for Statistical Computing.

Rad, M. S., Martingano, A. J., \& Ginges, J. (2018). Toward a psychology ofHomo sapiens: Making psychological science more representative of the human population. Proceedings of the National Academy of Sciences, 115(45), 1140111405. doi: 10.1073/pnas.1721165115

Rafanelli, C., Offidani, E., Gostoli, S., \& Roncuzzi, R. (2012). Psychological correlates in patients with different levels of hypertension. Psychiatry Research, 198(1), 154-160. doi: 10.1016/j.psychres.2011.09.014

Redina, O. E., \& Markel, A. L. (2018). Stress, Genes, and Hypertension. Contribution of the ISIAH Rat Strain Study. Current Hypertension Reports, 20(8). doi: 10.1007/ s11906-018-0870-2

Ruiz, J.-F. M. (2005). Los factores definitorios de los grandes grupos de edad de la población: Tipos, subgrupos, umbrales. Revista Electrónica de Geografía y Ciencias Sociales, 9(190). Recuperado de http://revistes.ub.edu/index.php/ ScriptaNova/article/view/901/878

Rutledge, T., \& Hogan, B. E. (2002). A Quantitative Review of Prospective Evidence Linking Psychological Factors With Hypertension Development. Psychosomatic Medicine, 64(5), 758-766. doi: 10.1097/01.psy.0000031578.42041.1c

Sahraian, A., Mokhtari, M., Moaref, A., Rezaee, V., Moghimi, E., \& Mani, A. (2015). Hypertensive patients and normotensive individuals: Differences in anger inventory. International Cardiovascular Research Journal, 9(4), 216219. doi: $10.17795 /$ icrj-9(4)216

Sanz, J. (2014). Recomendaciones para la utilización de la adaptación española del Inventario de Ansiedad de Beck (BAI) en la práctica clínica. Clínica y Salud, 25(1), 39-48. doi: $10.5093 / \mathrm{cl} 2014 \mathrm{a} 3$

Sanz, J., García-Vera, M. P., Espinosa, R., Fortún, M., Magán, I., \& Segura, J. (2010). Psychological Factors Associated with Poor Hypertension Control: Differences in Personality and
Stress between Patients with Controlled and Uncontrolled Hypertension. Psychological Reports, 107(3), 923-938. doi: 10.2466/09.15.20.pr0.107.6.923-938

Sanz, J., García-Vera, M. P., Fortún, M., \& Espinosa, R. (2005, julio). Desarrollo y propiedades psicométricas de una versión breve española del Inventario para la Depresión de Beck-II (BDI-II). Comunicación presentada en el $\mathrm{V}$ Congreso Iberoamericano de Evaluación Psicológica, Buenos Aires (Argentina), 1-2 de julio.

Siegel, J. M. (1986). The multidimensional anger inventory. Journal of Personality and Social Psychology, 51(1), 191200. doi: 10.1037/0022-3514.51.1.191

Soboka, M., Gudina, E. K., \& Tesfaye, M. (2017). Psychological morbidity and substance use among patients with hypertension: a hospital-based cross-sectional survey from South West Ethiopia. International Journal of Mental Health Systems, 11(1). doi: 10.1186/s13033-016-0108-0

Sorsdahl, K., Sewpaul, R., Evans, M., Naidoo, P., Myers, B., $\&$ Stein, D. J. (2016). The association between psychological distress, alcohol use and physical non-communicable diseases in a nationally representative sample of South Africans. Journal of Health Psychology, 23(4), 618-628. doi: 10.1177/1359105316642832

Spielberger, C. D. (1991). State-Trait Anger Expression Inventory manual. Odessa, FL: Psychological Assessmante Resources.

Suls, J. (2017). Toxic Affect: Are Anger, Anxiety, and Depression Independent Risk Factors for Cardiovascular Disease? Emotion Review, 10(1), 6-17. doi: $10.1177 / 1754073917692863$

Suls, J., \& Bunde, J. (2005). Anger, Anxiety, and Depression as Risk Factors for Cardiovascular Disease: The Problems and Implications of Overlapping Affective Dispositions. Psychological Bulletin, 131(2), 260-300. doi: 10.1037/0033-2909.131.2.260

Tabachnick, B. G., \& Fidell, L. S. (2007). Using Multivariate Statistics. Nueva York, EE. UU.: Pearson Education.

Tel, H. (2013). Anger and depression among the elderly people with hypertension. Neurology, Psychiatry and Brain Research, 19(3), 109-113. doi: 10.1016/j.npbr.2013.05.003

Tikhonoff, V., Hardy, R., Deanfield, J., Friberg, P., Kuh, D., Muniz, G., ... Richards, M. (2014). Symptoms of anxiety and depression across adulthood and blood pressure in late middle age. Journal of Hypertension, 32(8), 1590-1599. doi: 10.1097/hjh.0000000000000244

Tully, P. J., Peters, R., Pérès, K., Anstey, K. J., \& Tzourio, C. (2018). Effect of SSRI and calcium channel blockers on depression symptoms and cognitive function in elderly persons treated for hypertension: three city cohort study. International Psychogeriatrics, 30(9), 1345-1354. doi: 10.1017/s1041610217002903

Uceda, M. I., Sanz-Fernández, J., Espinosa-López, R., \& García-Vera, M. P. (2013). Perfil emocional y cognitivo de la hipertensión arterial esencial mantenida frente a la 
normotensión. Clínica y Salud, 24(2), 67-76. doi: 10.5093/ cl2013a8

Vos, T., Abajobir, A. A., Abate, K. H., Abbafati, C., Abbas, K. M., Abd-Allah, F., ... Abera, S. F. (2017). Global, regional, and national incidence, prevalence, and years lived with disability for 328 diseases and injuries for 195 countries, 1990-2016: a systematic analysis for the Global Burden of Disease Study 2016. The Lancet, 390(10100), 1211-1259. doi: 10.1016/s0140-6736(17)32154-2

Wang, H., Naghavi, M., Allen, C., Barber, R. M., Bhutta, Z. A., Carter, A., ... Coates, M. M. (2016). Global, regional, and national life expectancy, all-cause mortality, and causespecific mortality for 249 causes of death, 1980-2015: a systematic analysis for the Global Burden of Disease Study 2015. The Lancet, 388(10053), 1459-1544. doi: 10.1016/ s0140-6736(16)31012-1

Wilcox, R. (2012). Introduction to Robust Estimation and Hypothesis Testing. Nueva York, EE. UU.: Academic Press.

Wilcox, R. R., \& Keselman, H. J. (2003). Modern Robust Data Analysis Methods: Measures of Central Tendency. Psychological Methods, 8(3), 254-274. doi: 10.1037/1082-989x.8.3.254
Woodward, M. (2014). Epidemiology. Study Design and Data Analysis. Nueva York, EE. UU.: CRC Press.

Wu, J.-R., Cummings, D. M., Li, Q., Hinderliter, A., Bosworth, H. B., Tillman, J., \& DeWalt, D. (2018). The effect of a practice-based multicomponent intervention that includes health coaching on medication adherence and blood pressure control in rural primary care. The Journal of Clinical Hypertension, 20(4), 757-764. doi: 10.1111/jch.13265

Yan, L. L., Liu, K., Matthews, K. A., Daviglus, M. L., Ferguson, T. F., \& Kiefe, C. I. (2004). Psychosocial factors and risk of hypertension. The coronary artery risk development in young adults (CARDIA) study. ACC Current Journal Review, 13(2), 20-21. doi: 10.1016/j.accreview.2003.12.025

Yu, P., Wenpeng, C., Qi, C., Dong, W., Ting, A., \& Jin Y. (2015). Association between anxiety and hypertension: a systematic review and meta-analysis of epidemiological studies. Neuropsychiatric Disease and Treatment, 11, 1121-1130. doi: 10.2147/NDT.S77710

Zhang, Y., Chen, Y., \& Ma, L. (2018). Depression and cardiovascular disease in elderly: Current understanding. Journal of Clinical Neuroscience, 47, 1-5. doi: 10.1016/j. jocn.2017.09.022 